\title{
Nutraceutical Potentials of Carotino Oil and Its Effect on Blood Glucose and Antioxidant Enzymes in Streptozotocin Induced Diabetic Rats
}

\author{
M. Ramyasivaselvi ${ }^{1}$, S. Kowsalya ${ }^{1}$, S. Ramalingam ${ }^{2}$ and M. Ramanathan ${ }^{3}$ \\ 1. Department of Food Science and Nutrition, Avinashilingam Deemed University for Women, Coimbatore 641043, India \\ 2. PSG Institute of Medical Science and Research, Coimbatore 641004, India \\ 3. PSG College of Pharmacy, Coimbatore 641004, India
}

Received: April 25, 2011 / Published: November 20, 2011.

\begin{abstract}
Carotino oil, a blend of canola oil (80\%) and red palm fruit $(20 \%)$ is rich in nutraceuticals. Hence, the study aimed at evaluating the nutrient and nutraceutical potentials of carotino oil and to study the effect of carotino oil supplementation on blood glucose and antioxidant enzymes in streptozotocin induced diabetic rats. Initially, the physio-chemical properties and nutraceutical potentials of carotino oil were determined using standard procedures and acceptability of commonly consumed recipes was evaluated. Further, a group of twenty four albino rats (wistar strain) were selected and divided into four groups of six each (Groups A, B, C and D). The rats in groups B, C and D were induced diabetes by a single intraperitoneal administration of streptozotocin. Further the two groups $(\mathrm{C}$ and $\mathrm{D})$ of diabetic rats were given a low dose $(2.14 \mathrm{~mL} / \mathrm{kg})$ and high dose $(4.28 \mathrm{~mL} / \mathrm{kg})$ of carotino oil for a period of ten days. The rats in group A and B formed the control and diabetic control. The impact was studied in terms of changes in body weight, fasting blood glucose and antioxidant enzyme levels (superoxide dismutase and lipid peroxidase). Results indicated that carotino oil was rich in nutraceutical potentials and the impact of supplementation of carotino oil at low dose maintained glycemic control and improved antioxidant enzymes in streptozotocin induced diabetic rats.
\end{abstract}

Key words: Carotino oil, nutraceuticals, diabetic rats, glucose, antioxidant enzymes.

\section{Introduction}

Diabetes mellitus is a metabolic disorder resulting from deficiency of insulin leading to complications involving many organs [1]. Diabetes has become a leading killer disease in recent years and its prevalence is a "Basic Health Indicator" or for member states of the World Health Organization [2]. The prevalence of diabetes in all age group worldwide was estimated to be 2.8 per cent in 2000 and projected as 4.4 per cent in 2030 . The total number of people with diabetes is projected to rise from 171 million in 2000 to 366 million in 2030 [3]. According to the

Corresponding author: S. Kowsalya, Ph.D., associate professor, research fields: nutritional biochemistry, functional foods and nutraceuticals. E-mail: kowsiskk@yahoo.co.in.
International Diabetes Federation, India has been declared as the diabetes capital of the world with over million diabetics and this number is set to increase to 57 million by 2025. Diet has been recognized as a corner stone in the management of diabetes mellitus [ 4 , 5]. Diet therapy for type II diabetics is designed to help them improve or maintain their quality of life, achieve good blood glucose control and prevent and complications of diabetes. To achieve these goals, proper dietary management is advocated.

Fats and oil play an important role in maintaining the structure and function of a host's metabolic and immunological system and also provide a variety of benefits with their use in medical and designer foods [6]. Dietary supplements have traditionally been used 
to improve the nutritional status of individuals who cannot meet their nutritional needs by diet alone [7].

Red palm oil and canola oil as such are rich sources of antioxidants. Recently Carotino India Pvt. Ltd has launched its antioxidant rich premium red cooking oil "Carotino" which is manufactured in Malaysia. It is highly nutritious and 100 per cent natural oil and is a blend of canola oil ( 80 per cent) and red palm fruit extract (20 per cent). Carotino is high in pro-vitamin A (alpha and beta carotenes), rich in vitamin $\mathrm{E}$, a fine source of omega 3 and 6 essential fatty acids, a good source of lycopene and co-enzyme Q10, provitamin A, $\mathrm{E}$ and lycopene are powerful antioxidants which play a vital role in supporting the body's immune system, fighting diabetes, heart disease, stress, cancer and antiaging. It is also low in saturated fat and high in healthy monounsaturated fatty acid which helps lower bad (LDL) cholesterol and raise "good" (HDL) cholesterol. Unlike other cooking oil, carotino is not hydrogenated so it contains no harmful trans fats [8].

No literature is available on the use of "Carotino oil", its nutraceutical properties and its impact on glycemic control. Hence, the present study was undertaken with the specific objectives of determining the physio-chemical properties, exploring the nutrient and nutraceutical components present in carotino oil, conducting acceptability trials and studying the effect of carotino oil supplementation on blood glucose and antioxidant enzymes in streptozotocin induced diabetic rats.

\section{Materials and Methods}

The composition of Carotino oil is shown in Fig. 1.
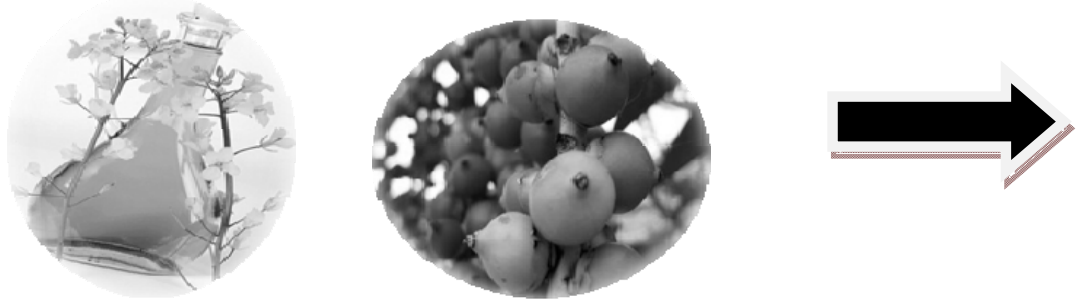

Canola oil $(80 \%)+$ Red palm fruit $(20 \%)=$ Carotino oil
The physio-chemical properties, nutrient and nutraceutical potentials of carotino oil were determined.

\subsection{Physical Properties}

The physical properties were studied in carotino oil in terms of colour, specific gravity, refraction index, moisture and viscosity. The colour of the oil was measured by Indian Standard. The specific gravity was measured by the method outlined by IS. Specific gravity which is defined as the ratio of the density of substances to the density of the reference substances at $25^{\circ} \mathrm{C}$ specific gravity. In general, either unsaturation of the fatty acid chain or increase in chain length of the fatty acid residues tends to increase the specific gravity. The index of refraction is the degree of deflection of a beam of light that occurs when it passes from one transparent medium to another. The refraction indices of fats and oils are often measured as specific gravity and refractive index, because they are rapidly and accurately determined and their usefulness in identification of adulterant substance in the testing of their purity. Refractive index determined by IS. Moisture determination is one of the most important and most widely used measurements in testing of foods [9]. Pure oils and fats contain practically no water. Moisture is one of the indicators of rancidity of fats and oils. Moisture was determined by IS. The resistance experienced by one layer of a liquid in moving over another layer is called "viscosity" [10]. Viscosity determines oil's relative thickness or resistance to flow. It was found by using Brookfield viscometer.

Fig. 1 Composition of carotino oil. 


\subsection{Chemical Properties}

The chemical properties of the carotino oil in terms of acid value, iodine value, saponification, unsaponifiable matter and turbidity test were determined. Acid value is an indicator used to determine the free fatty acid content of the oil which measures the extent to which hydrolysis has liberated the fatty acids from their ester linkage to the parent glyceride molecule. Iodine number is a measure of the extent of unsaturation of the fatty acids present in fats. When the iodine value decreases, the peroxide value increases. This is due to the formation of peroxides on the unsaturated linkages. The saponification number is defined as the number of milligrams of potassium hydroxide required to saponify one gram of fat or oil. If the fatty acids have long carbon chains and high molecular weight, the fat with low molecular weight fatty acids will consequently have a high saponification number [11]. Unsaponifiable matter in fats and oils include all unsaponifiable substances, wax and phytosterol compounds which are insoluble in water, but soluble in light petroleum spirit. The turbidity point is particularly sensitive to the presence of fatty acids and a correction factor must be introduced for these acids. Nevertheless, different oils show wide enough range of value so that the test has a value in the differentiation of some oils and in the detection of adulteration. The turbidity of oil was determined by cooling a mixture of it and a solvent in which it had limited solubility. The mixture was heated until complete solution occurs and then slowly cooled until detectable is known as the turbidity point at which turbidity first occurs. The temperature of oil at which turbidity was first detected is known as the turbidity point. All the chemical characteristics were determined by IS.

\subsection{Determination of Nutrients and Nutraceutical Compounds}

Determination of nutrient and nutraceutical compounds for the carotino was determined in terms of total fat, trans fatty acids, saturated fatty acids, omega 3 fatty acids, omega 6 fatty acids and vitamin E (gamma tocopherol). Total fat was measured by AOAC method [12]. Trans fats are formed during partial hydrogenation of oil. It was determined by IS method. Saturated fatty acid was determined by IS by using Gas Chromatography Flame Ionizing Detector. Omega 3 fatty acids and Omega 6 fatty acids were determined by IS by using Gas Chromatography Flame Ionizing Detector. Total carotene was determined by AOAC method [12] and beta and alpha carotene by HPLC Vitamin E (gamma tocopherol) was determined by IS. The fatty acid composition of carotino oil was determined by using gas chromatography and Flame Ionizing Detector.

\subsection{Acceptability Trials of Recipes Using Carotino Oil}

Organoleptic qualities play an important role in evaluating the quality of food product. Since carotino oil is new to the Indian food market, it was thought of interest to study the acceptability of commonly consumed recipes using carotino oil. A total of ten recipes namely sambar, tomato chutney, chappathi, omelette, poori, potato chips, dhal vadai, pappad, omapodi and vadagam were prepared using carotino oil. A numerical score card was used to measure the acceptability in terms of criteria appearance, colour, flavour, texture and taste with five scores for each criteria. A group of 20 semi trained panel members in the age group of 20-30 years evaluating the recipes. These scores given were excellent (5), very good (4), good (3), fair (2) and poor (1). The acceptability score was 25. For comparison purposes standard recipes using refined groundnut oil were prepared.

Since carotino oil is rich in nutrient and nutraceutical compounds, the investigator wanted to study the anti diabetic effect of carotino oil. Due to the non availability of literature, the ethical committee suggested the investigator to conduct the study in animal model initially. Therefore, the animal experiment was conducted in the following steps. 


\subsection{Effect of Carotino Oil on Blood Glucose and} Antioxidant Status in Streptozotocin Induced Diabetic Rats

\subsubsection{Selection of Animals}

The study was performed on matured 24 normoglycemic wistar strain albino rats, weighing 150-230 g, which were separately housed in cages. Animals were maintained in a room at $23 \pm 2{ }^{\circ} \mathrm{C}$, humidity 45 percent to 55 percent with a fixed $12 \mathrm{hr}$ artificial light period. Food and water were given ad libitum. Rats were fed with standard rodent diet until the initiation of treatment. All animals received human care, as outlined in the guide for the care and use of laboratory animals. The study was ethically approved by the Institutional Animal Ethics Committee of (No: 158/99 CPCSE), PSG Institute of Medical Science and Research (PSG IMS \& R) Coimbatore, Tamil Nadu, India.

\subsubsection{Grouping of Animals}

Twenty four adult wistar strain albino rats were randomly assigned into four groups of six each. Group A served as control, while Group B, C and D were the streptozotocin treated groups. Group B served as diabetic control, group $\mathrm{C}$ served as low dose and group D served as high dose. The grouping of albino rats is given in Table 1.

\subsubsection{Induction of Streptozotocin Induced Diabetes}

Streptozotocin (STZ; N-nitro derivative of glucosamine) is a naturally occurring, broad spectrum antibiotic and cyto-toxic chemical that causes degeneration of Langerhans of islets of beta cells in mammals. Induction of experimental diabetes in rats using streptozotocin is very convenient and simple to use. Streptozotocin (STZ) was used to induce diabetes

Table 1 Grouping of albino rats.

\begin{tabular}{lll}
\hline Groups & Treatment & No. of animals \\
\hline Group A-Control & No supplement & 6 \\
Group B-Diabetic control & No supplement & 6 \\
Group C & Low dose & 6 \\
Group D & High dose & 6 \\
Total & & 24 \\
\hline
\end{tabular}

in albino rats. Streptozotocin was freshly prepared by dissolving STZ in citrate buffer ( $\mathrm{pH}$ 4.5). The rats were initially fasted for $12 \mathrm{hr}$ and quickly given a single intraperitoneal administration of streptozotocin (45 $\mathrm{mg} / \mathrm{kg}$ of body weight). Diabetes mellitus was experimentally induced in groups B, C and D. After 72 $\mathrm{hr}$ of streptozotocin injection, the rats with plasma glucose more than $180 \mathrm{mg} / \mathrm{dL}$ were considered as diabetic rats and used for further experiment. Clinically, symptoms of diabetes were clearly seen in rats within 2-4 days following single intravenous or intraperitoneal injection of $60 \mathrm{mg} / \mathrm{kg}$ STZ. Thus, diabetes mellitus was induced in group B, C and D animals.

\subsubsection{Dosing Regimen}

The maximum single human dose of carotino oil recommended as per FDA is $15 \mathrm{~mL} /$ day [13]. The human dose was converted into animal dose as per the standard Body Surface Area (BSA) method. Accordingly, the dosage arrived for low dose was 2.14 $\mathrm{mL} / \mathrm{kg}$, high dose was $4.28 \mathrm{~mL} / \mathrm{kg}$ body weight of rats. The calculated dosage was adjusted to the mean body weight of each group of rats.

\subsubsection{Dosing Route}

Diabetic animals were divided into three groups of six animals each. One group formed the diabetic control which received no treatment. Another two groups of animals were administered with low dose $(2.14 \mathrm{~mL} / \mathrm{kg})$ and high dose $(4.28 \mathrm{~mL} / \mathrm{kg})$ of carotino oil orally for a period of ten days. At the end of the treatment period, overnight fasted rats were anesthetized under light ether and blood samples were collected from retro orbital plexus.

\subsubsection{Biochemical Analysis}

Biochemical analysis was carried out in blood samples for the following parameters.

\subsubsection{Estimation of Fasting Blood Glucose}

Fasting plasma glucose was measured by using E-choline Glucose kit (Merck) based on glucose oxidase method in auto analyzer.

\subsubsection{Antioxidant Enzymes Measurement}

(1) Superoxide Dismutase (SOD) 
Superoxide Dismutase (SOD) was assayed by the method of Kakkar et al. [14]. This was based on the $50 \%$ inhibition of the formation of NADH-phenazine methosulfate nitro blue tetrazolium formazan at 520 $\mathrm{nm}$ in a spectrophotometer.

\section{(2) Lipid Peroxidase}

Lipid peroxidase was estimated by measurement of thiobarbituric acid reactive substances (TBARS) in plasma by the method of Yagi [15]. The pink chromogen produced by the reaction of thiobarbituric acid with malondialdehyde, a secondary product of lipid peroxidation was estimated at $532 \mathrm{~nm}$ in a spectrophotometer.

\subsubsection{Statistical Analysis}

The data were analysed by one way Analysis of Variance (ANOVA) followed by Duncan's Multiple Range Test (DMRT), to determine the significant difference between the groups.

\section{Results and Discussion}

\subsection{Physical Characteristics of Carotino Oil}

Table 2 represents the physical characteristics of carotino oil.

The colour value represents the pigments present in the oil. Since carotino is a blend of canola oil $(80 \%)$ and red palm fruit $(20 \%)$, the colour is assumed from the natural components present in the oil. The colour value was 97 (Yellow +5 Red) which is similar to the value reported by "Carotino India Private Limited" [16]. The specific gravity measure was used to find the edibility of the oil. The specific gravity, refractive index, moisture and viscosity were similar to the data reported by "Carotino India Private Limited" [16]. While the refractive index of carotino oil and canola oil were similar in the range of 1.46-1.47, the specific gravity and viscosity of carotino oil was less than canola oil [16].

\subsection{Chemical Characteristics of Carotino Oil}

Table 3 represents the chemical characteristics of carotino oil.
Table 2 Physical characteristics of carotino oil.

\begin{tabular}{lc}
\hline Physical properties & Carotino oil \\
\hline Colour & 97 \\
Specific gravity & 0.918 \\
Refractive index & 1.46 \\
Moisture & 0.1 \\
Viscosity & 131 \\
\hline
\end{tabular}

Table 3 Chemical characteristics of carotino oil.

\begin{tabular}{lc}
\hline Chemical properties & Carotino oil \\
\hline Acid value & 0.06 \\
Iodine number & 109 \\
Saponification number & 198 \\
Unsaponifiable matter & 0.4 \\
Turbidity test & - \\
\hline
\end{tabular}

Regarding the chemical properties, the acid value of oils represents the free fatty acid content of oil. According to the act of Prevention of Food Adulteration in India [17], the maximum limit of free fatty acid in edible refined oils was 1 to 3 per cent. The acid value of carotino was 0.06 which was within the recommendations of Prevention of Food Adulteration. The acid value of canola oil was in the range of 0.4-1.2. The unsaponifiable matter in carotino was 0.4 whereas is canola, it was in the range of 0.5-1.2 and for red palm oil, it was not more than 1.2 per cent [18].

\subsection{Nutrient and Nutraceutical Components of Carotino Oil}

Table 4 gives the nutrient and nutraceutical components of carotino oil.

Carotino oil contains valuable nutrients and has the potential to be considered as a functional food. Carotino is high in antioxidant pro-vitamin A (alpha and beta carotenes) and also a good source of vitamin E (26 mg), high in omega 3 (6.6 g), omega 6 fatty acids (20 g), rich in MUFA (52.5 g), PUFA (25.3 g) and energy $(812 \mathrm{kcal})$. Other nutrients like protein, carbohydrate, sugar, fibre, sodium and cholesterol were found to be absent.

\subsection{Acceptability Trials of Recipes Prepared Using Carotino Oil}

Table 5 gives the overall acceptability scores of standard and carotino recipes. 
Table 4 Nutrient and nutraceutical components of carotino oil.

\begin{tabular}{lc}
\hline Nutrient and nutraceutical compounds & Carotino oil \\
\hline$\beta$-carotene (mg) & 5.3 \\
Alpha carotene (mg) & 4.1 \\
Total fat (g) & 92 \\
Trans fat acids (g) & $<0.5$ \\
Saturated fatty acids (g) & 14.3 \\
Omega-3 fatty acids (g) & 6.6 \\
Omega-6 fatty acids (g) & 20.0 \\
Vitamin E (mg) tocopherol and tocotrienol & 26 \\
Monounsaturated fat (g) & 52.5 \\
Polyunsaturated fat (g) & 25.3 \\
Energy (kcal) & 812 \\
Protein, carobohydrate, sugar, fibre, sodium and - \\
cholesterol
\end{tabular}

Table 5 Overall acceptability scores of standard and carotino recipes.

\begin{tabular}{lll}
\hline \multirow{2}{*}{ Recipes } & \multicolumn{2}{c}{ Over all acceptability (25) } \\
\cline { 2 - 3 } & Standard recipes & Carotino recipes \\
\hline Tomato chutney & $22.6 \pm 2.30$ & $22.95 \pm 2.37$ \\
Sambar & $22.75 \pm 2.40$ & $23.5 \pm 2.09$ \\
Poori & $22.45 \pm 2.43$ & $22.45 \pm 2.43$ \\
Potato chips & $21.5 \pm 2.15$ & $22.75 \pm 2.44$ \\
Chappathi & $21.55 \pm 2.81$ & $23 \pm 1.9$ \\
Omelette & $22.95 \pm 2.91$ & $24.45 \pm 1.14$ \\
Dhal vadai & $23.1 \pm 2.24$ & $22.65 \pm 2.90$ \\
Omapodi & $22.6 \pm 3.5$ & $22.8 \pm 3.73$ \\
Pappad & $21.95 \pm 3.28$ & $21.9 \pm 2.53$ \\
Vadagam & $21.95 \pm 3.36$ & $22.25 \pm 2.48$ \\
\hline
\end{tabular}

The acceptability trials of commonly consumed recipes using caotino oil in comparison to the standard preparations proved that carotino recipes ranked higher and were more acceptable. Hence carotino oil may be used in different methods of preparation in India. The recipes would not only add taste and colour, but also provide important nutraceuticals beneficial to the human body.
3.5 Effect of Carotino Oil on Blood Glucose and Antioxidant Enzymes in Streptozotocin Induced Diabetic Rats

Carotino oil was given to streptozotocin induced diabetic rats in two doses namely low dose and high dose. The impact was studied in terms of body weight, blood glucose and antioxidant enzymes such as superoxide dismutase (SOD) and lipid peroxidase (LPO).

\subsubsection{Body Weight of the Selected Albino Rats}

Table 6 and Fig. 2 give the changes in mean body weight $(\mathrm{kg})$ of the selected albino rats.

The initial body weight was similar among the four groups. In control, there was not much difference in the average body weight. Diabetic rats in general showed reduction in weight compared to control at the end of the treatment period. Group C and D rats after administration of low and high dose of carotino oil showed mild increments in weight compared to group D which showed reduction. According to Alireza [3], diabetic rats showed significant weight loss, polyphagia and polydipsia compared with control at the end of the treatment period. In general, the changes in body weight of rats showed significant difference at one per cent level.

\subsubsection{Fasting Blood Glucose Levels}

Table 7 and Fig. 3 give the mean blood glucose levels of the albino rats.

The initial fasting blood glucose was similar among the three groups. In groups $\mathrm{B}, \mathrm{C}$ and $\mathrm{D}$, the streptozotocin induced diabetic rats showed higher levels of fasting blood glucose levels. After ten days, no change in fasting glucose was noted in group I rats (normal). The administration of carotino oil to group $\mathrm{C}$

Table 6 Changes in mean body weight of the selected albino rats (kg).

\begin{tabular}{lllll}
\hline Groups & Initial (Mean \pm SD) & Final (Mean \pm SD) & Difference & $F$ value \\
\hline Group A (Control) & $206.5 \pm 8.36$ & $206.67 \pm 8.16$ & $0.166(\mathrm{~b})$ & \\
Group B (Diabetic control) & $188.67 \pm 7.94$ & $186.67 \pm 7.52$ & $-2.0(\mathrm{a})$ & $11.60^{* *}$ \\
Group C (Low dose) & $186.67 \pm 5.12$ & $187.83 \pm 5.26$ & $1.16(\mathrm{a})$ & \\
Group D (High dose) & $188 \pm 5.05$ & $190.33 \pm 5.35$ & $2.33(\mathrm{a})$ & \\
\hline
\end{tabular}

\footnotetext{
** Significant at $1 \%$ level. Mean followed by a common letter are not significantly different at the $5 \%$ level by DMRT.
} 
Nutraceutical Potentials of Carotino Oil and Its Effect on Blood Glucose and Antioxidant Enzymes in Streptozotocin Induced Diabetic Rats

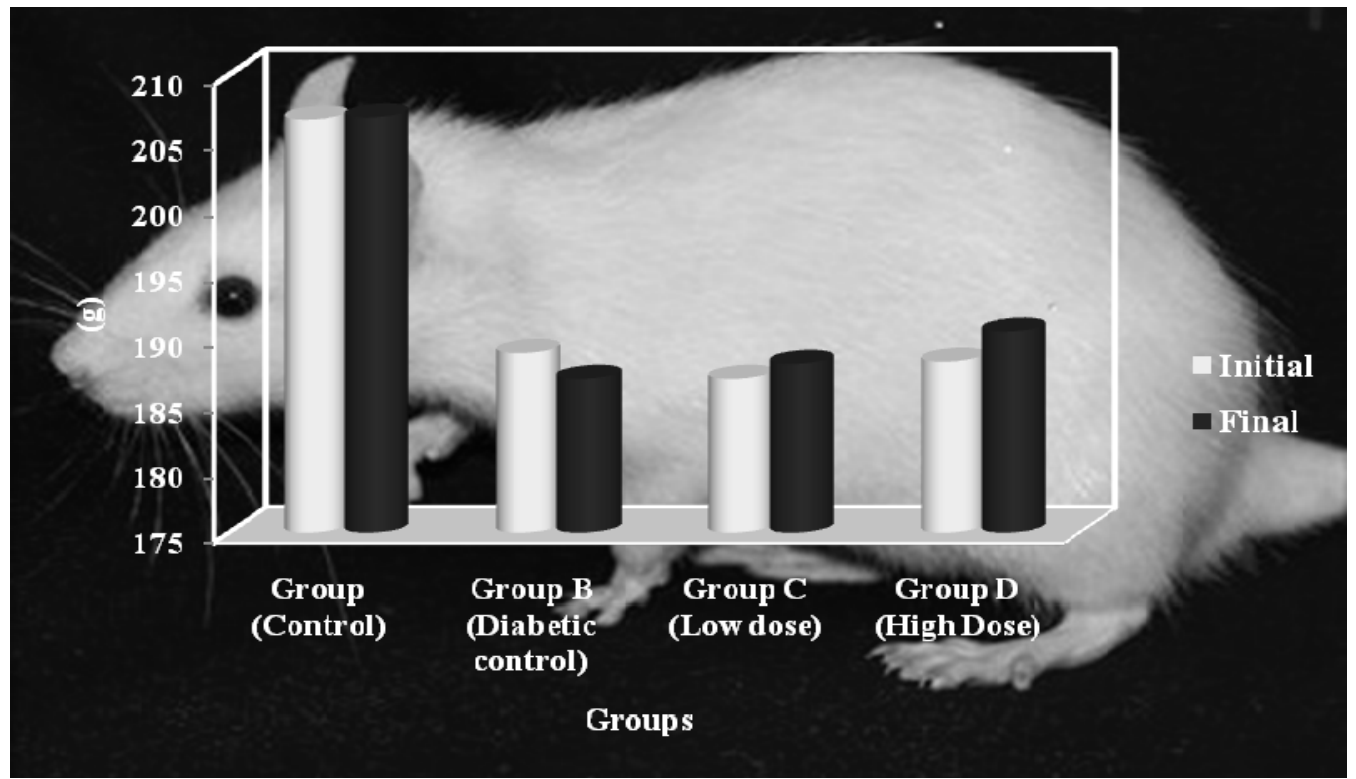

Fig. 2 Changes in mean body weight of the selected albino rats.

Table 7 Changes in mean fasting blood glucose levels (mg/dL).

\begin{tabular}{lcclc}
\hline Groups & Initial (Mean \pm SD) & Final (Mean \pm SD) & Difference & $F$ value \\
\hline Group A (Control) & $76.75 \pm 6.58$ & $76.83 \pm 6.67$ & $+0.08(\mathrm{a})$ & \\
Group B (Diabetic control) & $337.0 \pm 8.47$ & $339.0 \pm 8.94$ & $+1.5(\mathrm{c})$ & \\
Group C (Low dose) & $338.83 \pm 10.4$ & $337.67 \pm 9.15$ & $-1.16(\mathrm{c})$ & $1253.80^{* *}$ \\
Group D (High dose) & $339.5 \pm 7.44$ & $305.67 \pm 9.83$ & $-33.83(\mathrm{~b})$ & \\
\hline
\end{tabular}

** Significant at $1 \%$ level. Means followed by a common letter are not significantly different at the $5 \%$ level by DMRT.

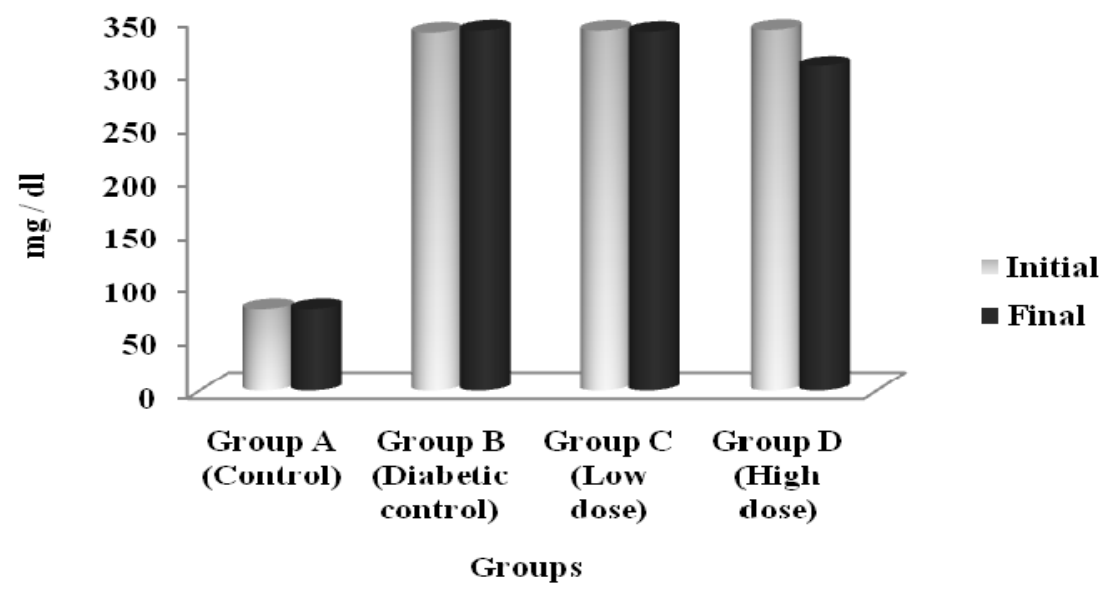

Fig. 3 Changes in mean fasting blood glucose levels.

brought about a minor reduction in blood glucose level and it may be considered that low dose of carotino oil maintained the blood glucose level. Whereas in group $\mathrm{D}$ given high dose brought about a significant reduction to the level of $33 \mathrm{mg}$. The differences in glucose levels among the four groups were found to be significant at one per cent level by ANOVA. Parisa [19] stated that there was a significant elevation in glucose after 48 hours administration of STZ. After 10 days, no significant elevation of glucose was observed in 
control groups. Diets rich in monounsaturated fat obtained from canola oil, almonds, virgin olive oil, peanut oil and/or peanut butter have been shown to help control blood glucose in free-living people with or without type II diabetes [20].

\subsubsection{Antioxidant Enzymes}

Table 8 and Fig. 4 show that the changes in mean superoxide dismutase levels of the selected albino rats.

Superoxide dismutase is considered a primary enzyme since it is involved in the direct elimination of reactive oxygen species [21]. The initial level of superoxide dismutase was lower in diabetic rats compared to control group. The administration of carotino oil to groups $\mathrm{C}$ and $\mathrm{D}$ increased the levels of the enzyme. Low dose showed better increment in superoxide dismutase than high dose. Non supplemented diabetic control showed a decrease in
SOD level. In general, the changes in SOD levels among the four groups were found to be significant at one per cent by ANOVA.

Fig. 5 gives the changes in mean lipid peroxidase (LPO) level of the animals.

The initial lipid peroxide concentration level was similar among the diabetic group of rats. Lipid peroxide (LPO) level was elevated in diabetic rats as compared to normal rats. Ragavan et al. [22] showed that in liver and kidney, lipid peroxide (LPO) levels were elevated significantly $(P<0.05)$ in diabetic rats as compared to normal rats. Group $\mathrm{C}$ and $\mathrm{D}$ rats treated with carotino oil showed reduction in the LPO concentration. This proves the nutraceutical potentials of carotino oil. In general, the changes in lipid peroxide levels among the four groups were found to be significant at one per cent level.

Table 8 Changes in mean superoxide dismutase levels ( $\mathrm{U} \mathrm{mg} / \mathrm{protein})$.

\begin{tabular}{lllll}
\hline Groups & Initial $($ Mean $\pm \mathrm{SD})$ & Final $($ Mean $\pm \mathrm{SD})$ & Difference $(\mathrm{mg} / \mathrm{dL})$ & $F$ value \\
\hline Group A (Control) & $4.42 \pm 0.08$ & $4.43 \pm 0.06$ & $0.003(\mathrm{~d})$ & \\
Group B (Diabetic control) & $2.83 \pm 0.12$ & $2.566 \pm 0.07$ & $-0.266(\mathrm{a})$ & $434.54 * *$ \\
Group C (Low dose) & $2.21 \pm 0.12$ & $3.296 \pm 0.12$ & $1.085(\mathrm{c})$ & $0.67(\mathrm{~b})$ \\
Group D (High dose) & $2.36 \pm 0.28$ & $3.03 \pm 0.09$ & \\
\hline
\end{tabular}

** Significant at $1 \%$ level. Means followed by a common letter are not significantly different at the $5 \%$ level by DMRT.

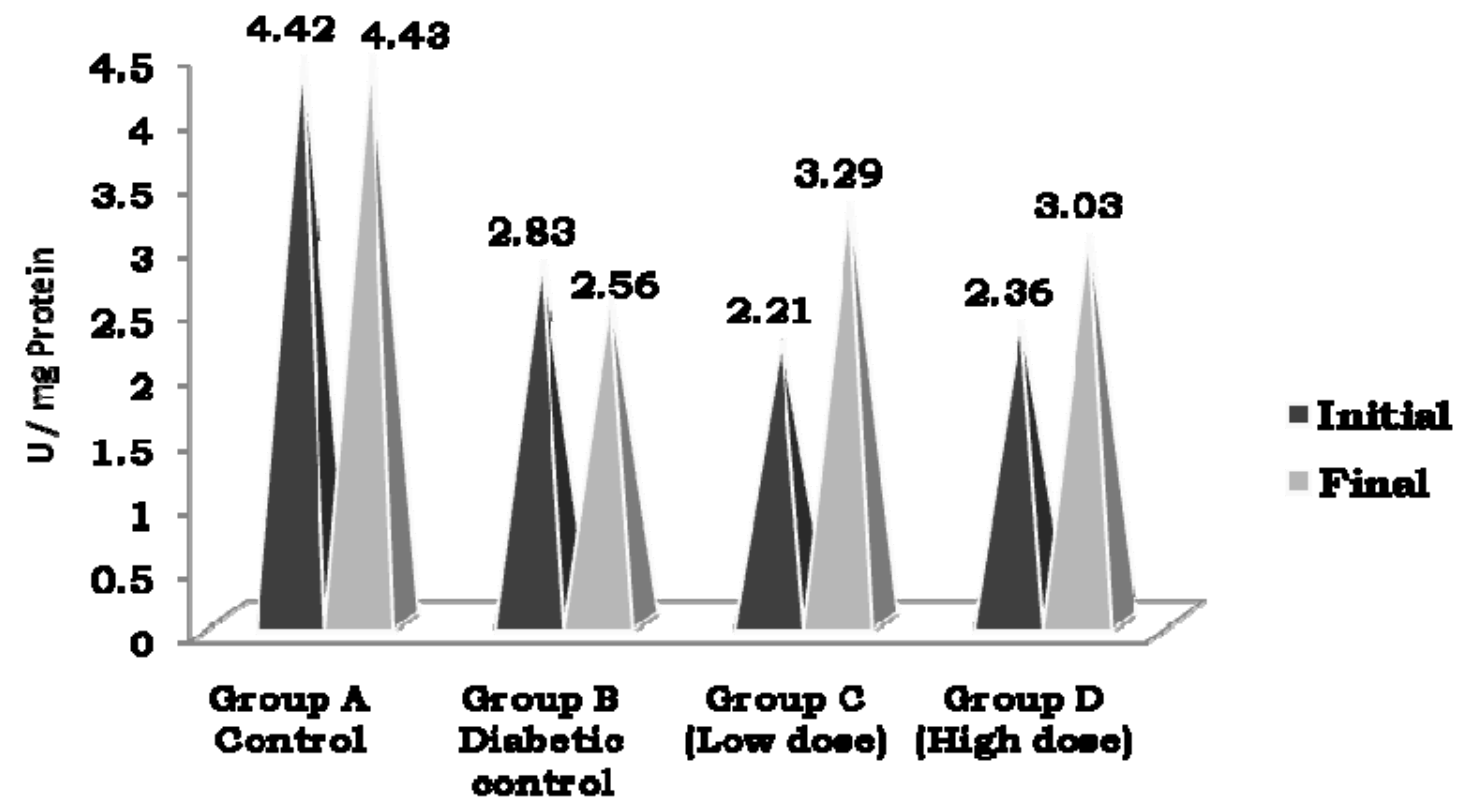

\section{GROUPS}

Fig. 4 Changes in mean superoxide dismutase levels. 


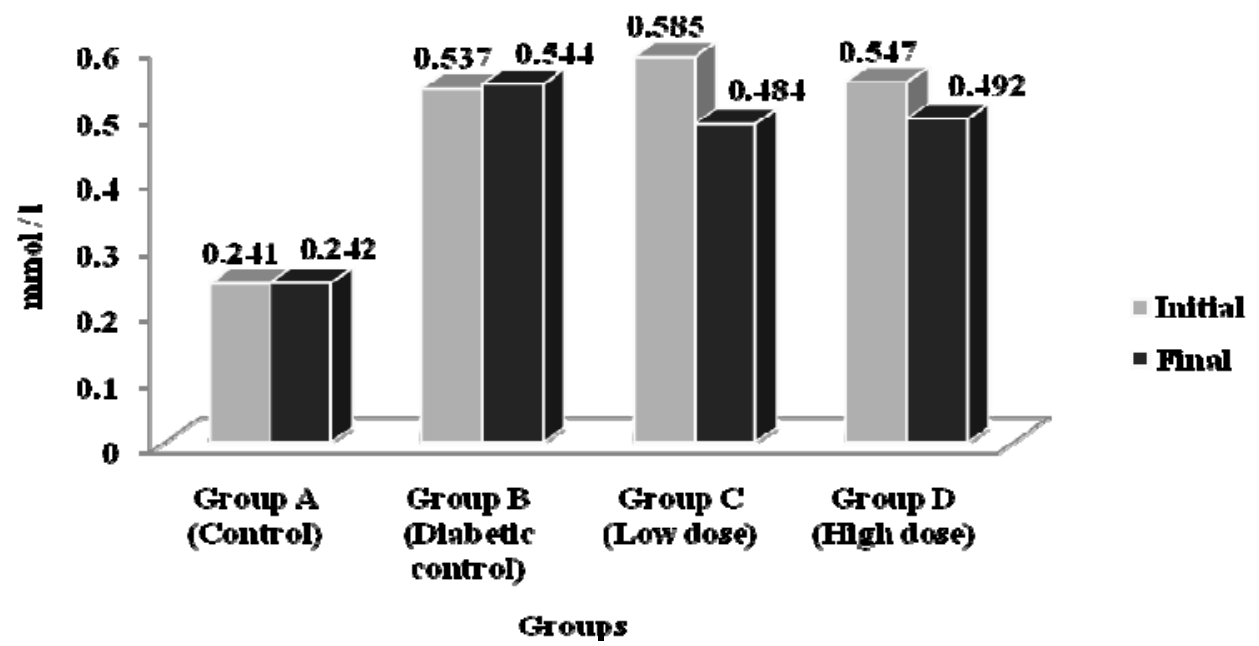

Fig. 5 Changes in mean lipid peroxide levels.

\section{Conclusions}

From the foregoing results and discussions, it may be concluded that the carotino oil possess high nutrient and nutraceutical potentials. It is evident in terms of vitamin E, A, Omega 3 and Omega 6 fatty acids. The recipes prepared using carotino oil was found to be highly acceptable. Further, the effect of carotino oil on streptozotocin induced diabetic rats showed that low dose brought about significant improvement in antioxidant enzymes and maintained blood glucose level. High dose of carotino though, brought reduction in glucose level it did not produce acute hypoglycemia. Hence, further studies on carotino supplementation in healthy volunteers and diabetic subjects are recommended.

\section{Acknowledgment}

The authors thank Carotino India Private Limited, Pune, India for sponsoring "Carotino oil" for the entire research study.

\section{References}

[1] M. Chandrashekhar, S. Sultanpur, N. Sathyanaranyana, R. Sreenivasa, K. Vasanth, Influence of jenofibrate on the pharmaco dynamics of gliclazide in rats and rabbits, Research Journal of Pharmaceutical Biological and Chemical Sciences 1 (2010) 320-329.

[2] M. Majeed, L. Pragash, Diabetes Management the Therapeuctic Role of Ayurvedic Herbs, 2005, available online at: www.mail.google.com/mail(?vi=1\&view=atl \& view $=$ att $8^{\text {th }}=1261$ e 3 daa $895 f 61$ a $\&$ attid= $=0.2 \&$ disp=va.

[3] A. Nakhaee, M. Bokaeian, M. Sardvani, A. Farhangi, A. Akbarzadeh, Attenuation of oxidative stress in streptozotocin induced diabetic rats by eucalyptus globules, Indian Journal of Clinical Biochemistry 24 (2009) 419.

[4] T. Sathyanarayana, P. Dwarakanadha Reddy, D. Swarnalatha, A. Mathews, Hypoglycemic effect of a poly herbal extracts on normal and streptozotocin induced diabetic rats, International Journal of Pharmacy and Pharmaceutical Sciences 1 (2010) 3.

[5] M. Yaheya, M. Ismail, N. Asses, M. Zakriya, Role of spices in diabetes mellitus, Research Journal of Pharmaceutical, Biological and Chemical Sciences 3 (2010) 3.

[6] Nutrition Fact Sheet, 2006, available online at: www.canola.org.

[7] Mary, Dietary Supplements Health and Education, 2010, pp. 103-417.

[8] Carotino India Private Limited, "New Products", Processed Food Industry 43 (2009) 64.

[9] Makower, Physical and chemical changes in vegetable oil blends, Journal of American Oil Chemists Society 1 (1950) 7-9.

[10] A.C. Deb, Fundamentals of Biochemistry, New Central Book Agency Private Limited, 2006, p. 36.

[11] Hoggland, Interactive effects of dietary proteins and dietary lipids on plasma and liver lipid profiles, Journal of American oil Chemists Society 68 (2003) 956-959.

[12] AOAC, Official Methods and Recommended Practices of American Oil Chemists Society, Manual of Policies and Procedures, 2006.

[13] Available online at: http://www.carotino.co.uk.

[14] P.S. Kakkar, B.B. Das, P.N. Viswanathan, A modified spectrophotometric assay of superoxide dismutase, Indian 

Antioxidant Enzymes in Streptozotocin Induced Diabetic Rats

Journal of Biochemistry and Biophysics 21 (1984) 130-132.

[15] K. Yagi, Lipid peroxides and human disease, Chem. Phys. Lipids 45 (1978) 337-351.

[16] Carotino Indian Privated Limited, available online at: www.carotino.co.uk.

[17] Prevention of Food Adulteration Act, Laboratory Manual, 1954, p. 54.

[18] Health Canada, 2005, available online at: http://www.canolainfo.org/canola/index.php.

[19] P. Sarkhail, S. Rahmainpour, S. Fadyevatan, A. Mohammadirad, G. Dehghan, G. Amin, et al., Antidiabetic effect of Phlomis anisodonata: effects on hepatic cells lipid peroxidation and antioxidant enzymes in experimental diabetes, Pharmacological Research the Official Journal of the Italian Pharmacological Society 2 (2007) 11.

[20] E. Ros, Dietary cis-monounsatured fatty acids and metabolic control in type II diabetes, American Journal of Clinical Nutrition 78 (2003) 617S-625S.

[21] S.A. Moussa, Oxidative stress in diabetes mellitus, Romanian Journal of Biophysics 18 (2008) 225-236.

[22] V. Ragavan, J. Kalaivanishailaja, N. Nanlini, Effect of Terminalia arjuna stem bark on antioxidant status in liver and kidney of alloxan diabetic rats, Indian Journal of Phamacoloy 50 (2006) 133-142. 